\title{
Epsilon near-zero all-optical terahertz modulator
}

Cite as: Appl. Phys. Lett. 117, 111101 (2020); https://doi.org/10.1063/5.0012206

Submitted: 30 April 2020 . Accepted: 03 September 2020 . Published Online: 14 September 2020

E. Alvear-Cabezón (D), T. Taliercio (D), S. Blin (D), R. Smaali, F. Gonzalez-Posada (D), A. Baranov, R. Teissier, and E. Centeno (D)
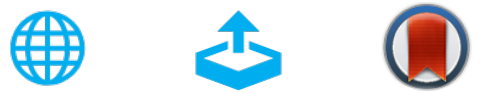

View Online

Export Citation

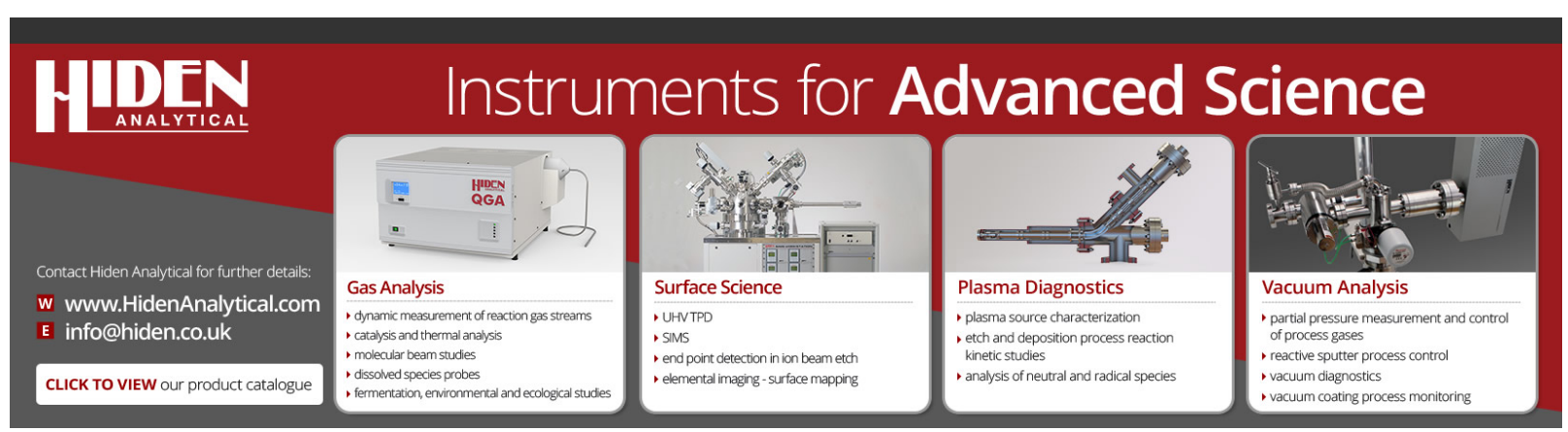




\title{
Epsilon near-zero all-optical terahertz modulator
}

\author{
Cite as: Appl. Phys. Lett. 117, 111101 (2020); doi: 10.1063/5.0012206 \\ Submitted: 30 April 2020 - Accepted: 3 September 2020 . \\ Published Online: 14 September 2020
}

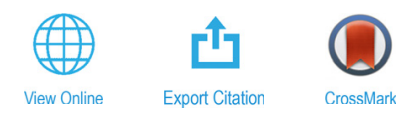

\author{
E. Alvear-Cabezón, ${ }^{1,2, a)}$ (D) T. Taliercio, ${ }^{2}$ (D) S. Blin, ${ }^{2}$ (D) R. Smaali, ${ }^{1}$ F. Gonzalez-Posada, ${ }^{2}$ (D) A. Baranov, ${ }^{2}$ R. Teissier, ${ }^{2}$ \\ and E. Centeno ${ }^{1, a)}$ (iD)
}

\author{
AFFILIATIONS \\ 'Université Clermont Auvergne, CNRS, SIGMA Clermont, Institut Pascal, F-63000 Clermont-Ferrand, France \\ ${ }^{2}$ IES, Univ Montpellier, UMR CNRS 5214, Montpellier, France
}

${ }^{a)}$ Authors to whom correspondence should be addressed: eduardo.alvear10@gmail.com and emmanuel.centeno@uca.fr

\begin{abstract}
We propose an efficient optically actuated $\mathrm{THz}$ modulator based on an ultrathin epsilon-near-zero (ENZ) slab photogenerated in an InAs semiconductor. We experimentally demonstrate a modulation depth of $90 \%$ at $1 \mathrm{THz}$ obtained with a continuous laser at irradiation lower than $10 \mathrm{~W} \mathrm{~cm}^{-2}$. Beyond the strong attenuation of the $\mathrm{THz}$ transmission provided by the ENZ absorption effect, we also report a broadband modulation of the $\mathrm{THz}$ waves from 1 to $10 \mathrm{THz}$. In addition, our experimental results show that the cut-off frequency of $3 \mathrm{~dB}$ attains $2 \mathrm{MHz}$ in the dynamic modulation regime.
\end{abstract}

Published under license by AIP Publishing. https://doi.org/10.1063/5.0012206

The development of the technology based on terahertz $(\mathrm{THz})$ electromagnetic radiation is crucial for many application domains such as security, medicine, and wireless communication. ${ }^{1-6}$ The demand for versatile $\mathrm{THz}$ components operating in the $\mathrm{THz}$ gap $(0.1-10 \mathrm{THz})$ is high. Artificial structures such as metasurfaces have been recently investigated to dynamically control $\mathrm{THz}$ waves. ${ }^{7-9}$ Thermal, mechanical, electrical, and optical actuations have been explored to reach an efficient and high speed modulation of $\mathrm{THz}$ signals. Comparison between these approaches can be found in several reviews, see, for example, Table I of Refs. 10 and 11. Among those approaches, all-optical devices have attracted great interest since they provide a high modulation depth. Photocarriers are generated within a semiconductor, such as silicon, GaAs, and Ge, when it is illuminated by a pump beam. This excess of carriers modifies the mobility and/or the conductivity of the material, which is probed by the $\mathrm{THz}$ signal. To improve the modulation depth and lower the pump power, 2D materials such as graphene have been deposited on such semiconductors. ${ }^{12}$ In that case, a modulation depth up to $94 \%$ has been reached but for a low modulation speed of $200 \mathrm{kHz}$ and in a broadband range lower than $1 \mathrm{THz} .{ }^{13}$ Comparison of these optically actuated THz modulators is reviewed in Refs. 11 and 14. Usually, the resonant behavior of metasurfaces limits the modulation of the $\mathrm{THz}$ signal over a wide broadband of frequency. Photo-generated metasurfaces have been developed to overcome this limitation. In that case, a pump laser induces a local refractive index modification into a semiconductor layer, leading to optically printed metasurfaces. ${ }^{15-21}$ The design is scaled according to the targeted $\mathrm{THz}$ frequency, which provides an optimal modulation. The main issue that prevents the implementation of alloptical THz modulators in the application domain concerns the high pump power required to modulate efficiently the $\mathrm{THz}$ signal. However, recent theoretical results suggest that Indium Arsenide (InAs) is a promising semiconductor to realize high $\mathrm{THz}$ modulation using a low pump power in the continuous-wave $(\mathrm{CW})$ regime. ${ }^{22,23}$ Recent studies demonstrate that perfect absorption also occurs within a slab when the real part of the permittivity approaches zero. It has been demonstrated that epsilon-near zero (ENZ) modes efficiently absorb the incoming radiation in an ultrathin slab, presenting very small optical losses. ${ }^{24-27}$ An additional coupling device is needed to excite such ENZ guided modes by using resonators or a prism in the Kretschmann-Raether configuration. ${ }^{28-30}$ Moreover, several works have demonstrated that the ENZ frequency can be dynamically varied with transparent conductive oxides (for example, indium tin oxide, ITO) or with doped semiconductors. ${ }^{28-30}$ In that case, an applied voltage modifies the electronic density, which, in turn, shifts the ENZ frequency. The maximum frequency shift of the absorption peak experimentally observed attains $20 \%$ for a modulation depth of about $20 \%$ and a $125 \mathrm{kHz}$ modulation rate. ${ }^{29}$ No matter how these approaches are implemented, the modulation of the $\mathrm{THz}$ signal depends on the strength of the coupling between the $\mathrm{THz}$ waves and the active material. This condition is usually reached by properly designing the metasurface (such as the size and the shape of the resonators). ${ }^{31,32}$ Finally, efficient $\mathrm{THz}$ modulators are generally complex systems, not always CMOS compatible and operates in a narrow frequency range and sometime at very low temperature. In this work, we 
propose a very simple all-optical $\mathrm{THz}$ modulator based on an InAs slab. We demonstrate a modulation depth up to $90 \%$ at $1 \mathrm{THz}$ for a low pump irradiance of $8.1 \mathrm{~W} \mathrm{~cm}^{-2}$ in the continuous-wave regime. The optically induced modification of the refractive index produces a broadband modulation of the $\mathrm{THz}$ transmission from $1 \mathrm{THz}$ to $10 \mathrm{THz}$. In addition, a modulation speed up to $2 \mathrm{MHz}$ is experimentally demonstrated in the pulse regime. The InAs semiconductor is an attractive material, compatible with the CMOS technology and operating at room temperature. ${ }^{33}$ In addition, the modulator is very compact since the thickness of the InAs slab is about $\lambda / 50$. This efficient absorption within an ultrathin slab is explained by the photogeneration of an ENZ material.

The sample used in this work is a free-standing InAs slab of $6 \mu \mathrm{m}$ thickness. Its preparation needs the following steps: a $15 \mu \mathrm{m}$ thick layer of an undoped InAs and a thin layer of $300 \mathrm{~nm}$ of AlAsSb (etchstop) are deposited by solid-source molecular beam epitaxy on a doped InAs substrate. After a partial polishing of the InAs substrate, the undoped InAs layer is stuck using an epoxy adhesive (ECCOBOND 144A) to a sample holder of copper covered by a thin gold layer. Then, the rest of the InAs substrate is chemically etched by a solution of citric acid diluted in hydrogen peroxide $\left[\mathrm{C}_{6} \mathrm{H}_{8} \mathrm{O}_{7}: \mathrm{H}_{2} \mathrm{O}_{2}\right.$ (2:1)]. The etching process stops on the AlAsSb layer thanks to the selective etching between AlAsSb and InAs. ${ }^{34}$ Finally, the AlAsSb layer is removed with a HF buffer and the InAs slab is progressively thinned by using a solution of phosphoric acid diluted in hydrogen peroxide and water $\left[\mathrm{H}_{3} \mathrm{PO}_{4}: \mathrm{H}_{2} \mathrm{O}_{2}: \mathrm{H}_{2} \mathrm{O}(2: 1: 1)\right]$ up to $6 \mu \mathrm{m}$. The InAs slab covers a $2 \mathrm{~mm}$ hole of the sample holder. The slab thickness is controlled by transmission experiments from 1 to $10 \mathrm{THz}$ (Fig. 1) using a Bruker Vertex 70 Fourier transform infrared (FTIR) spectrometer equipped with a water-cooled far-infrared light-source, a Si-beam splitter, and a cooled Si bolometer. The FTIR experiments are done under $N_{2}$ flux. All the spectra are numerically smoothed to remove absorption lines from residual gases other than $\mathrm{N}_{2}$ in the FTIR spectrometer. We used an $808 \mathrm{~nm}$ laser with a collimated beam, with a diameter larger than the hole of the sample holder and a tunable irradiance to photogenerate free carriers in the InAs slab. The transmission spectrum with null irradiance in Fig. 1 shows interference fringes allowing us to adjust our model using only the thickness as a

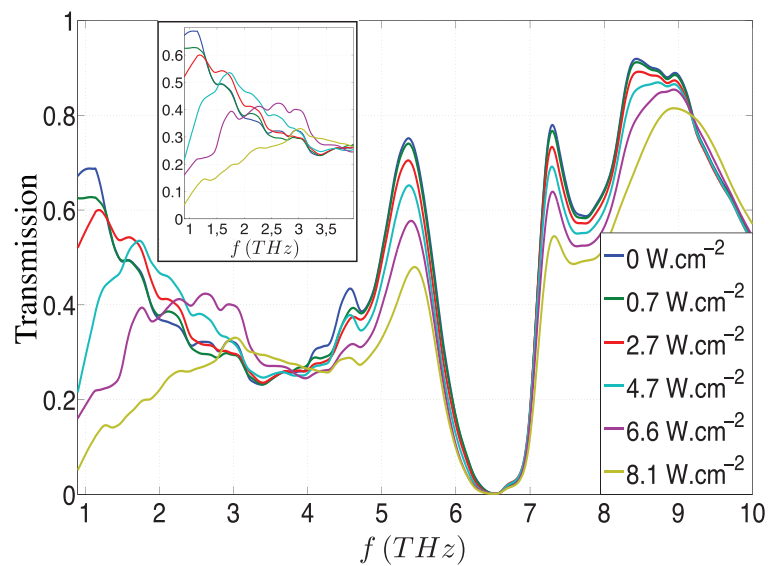

FIG. 1. Experimental transmission spectra for various pump irradiance values $\Phi_{0}:\{0,0.7,2.7,4.7,6.6,8.1\} \mathrm{W} \mathrm{cm}^{-2}$. The inset represents a zoom of the spectra. parameter. Without the laser pump, the transmission peaks originating from Fabry-Pérot (FP) resonances arise around 1, 5.5, 7.25, and 8.3 THz. The THz wave is reflected in the Reststrahlen phonon band in the range of 6-7 THz. Several modifications appear in the transmission spectrum as the laser pump irradiance denoted as $\Phi_{0}$ increases from 0 to $8.1 \mathrm{~W} \mathrm{~cm}^{-2}$. In the range of $1-4 \mathrm{THz}$, we observe a shift and a broadening of the main transmission peak. Besides, the transmission is substantially modulated (up to $30 \%$ ) at the FP resonances $(5.5,7.25$, and $8.3 \mathrm{THz}$ ) since the dielectric function is significantly modified.

The optical modulation of the $\mathrm{THz}$ signal for a very low pump irradiance is clearly demonstrated by representing the relative transmission modulation defined as $\left(T_{\text {off }}-T_{\text {on }}\right) / T_{\text {off }}$ where $T_{\text {off }}$ and $T_{\text {on }}$ are the transmission spectra when the pump is turned off $\left(\Phi_{0}=0\right)$ and on, respectively (Fig. 2). The maximal modulation depth attains $90 \%$ at $1 \mathrm{THz}$ for $\Phi_{0}=8.1 \mathrm{~W} \mathrm{~cm}^{-2}$. This very low pump irradiance produces a drastic change for the $\mathrm{THz}$ transmission that decreases from $69 \%$ to $7 \%$. This high modulation occurs for a wavelength fifty times larger than the InAs layer thickness. The modulation attains $42 \%, 32 \%$, and $27 \%$ at the respective FP resonances 5.5, 7.25, and 8.3 THz. The modulation is not considered in the Reststrahlen phonon band since the transmission is close to zero in this range independently of the pump power.

To understand these results, the permittivity of the InAs slab is modeled by combining a Lorentz model for the phonon resonances and a Drude model for the free-carrier effect,

$$
\varepsilon_{m}=\varepsilon_{\infty}\left(1-\frac{\omega_{p}(n)^{2}}{\omega(\omega+i \gamma)}+\frac{\omega_{L O}^{2}-\omega_{T O}^{2}}{\omega_{T O}^{2}-\omega^{2}-i \omega \Gamma}\right),
$$

where $\varepsilon_{\infty}=12.32, \omega_{L O}=45 \times 10^{12} \mathrm{rads}^{-1} \omega_{T O}=40 \times 10^{12} \mathrm{rad} \mathrm{s}^{-1}$ $\Gamma=0.56 \times 10^{12} \mathrm{rad} \mathrm{s}^{-1}$. The angular plasma frequency $\omega_{p}(n)$ for the Drude model is a function of the carrier density $n$,

$$
\omega_{p}(n)=\sqrt{n e^{2} /\left(m_{e f f} \varepsilon_{0} \varepsilon_{\infty}\right)} .
$$

The damping coefficient is $\gamma=e /\left(\mu \mathrm{m}_{\text {eff }}\right)$, where $m_{\text {eff }}=0.024 m_{e}$ is the electron's effective mass (with $m_{e}$ being the electron mass) and $\mu=24000 \mathrm{~cm}^{2} \mathrm{~V}^{-1} \mathrm{~s}^{-1}$ the electronic mobility.

The laser pump photogenerates electron-hole pairs that diffuse together within the InAs slab. This carrier density $n$ obeys to an

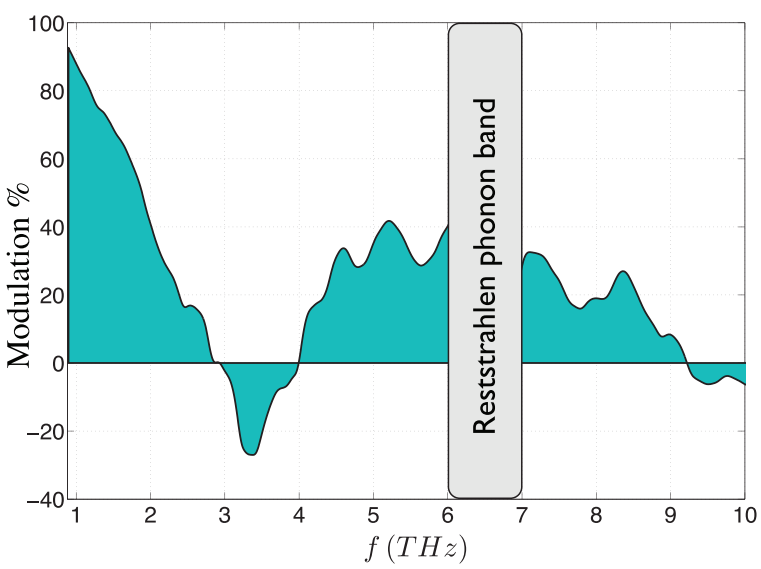

FIG. 2. Relative transmission modulation of an undoped $6 \mu \mathrm{m}$ thick InAs slab irradiated with a pump irradiance of $8.1 \mathrm{~W} \mathrm{~cm}^{-2}$ at room temperature. 
ambipolar transport equation that is solved following the calculations presented in Ref. 22. We consider an intrinsic doping $n_{0}=2 \times 10^{15} \mathrm{~cm}^{-3}$.

With this model, the permittivity derived from Eq. (1) is calculated when the pump switches from off to on (Fig. 3). Without the optical pumping, the real part of the permittivity vanishes for two ENZ frequencies: around the plasma frequency of $f_{p}=\omega_{p} / 2 \pi$ at $0.48 \mathrm{THz}$ and at the longitudinal optical phonon frequency of $f_{L O}=7.2 \mathrm{THz}$. When the pump is turned on $\left(\Phi_{0}=8.1 \mathrm{~W} \mathrm{~cm}^{-2}\right)$, the plasma frequency shifts to $f_{p}=1.75 \mathrm{THz}$. According to Eq. (2), the increase in the photogenerated carrier density causes the increase in the plasma frequency. Beyond the control over the plasma frequency allowed by the optical pump, the permittivity is globally modified at higher frequencies. Indeed, the Drude term in Eq. (1) introduces changes in both the real and imaginary parts that affect the overall permittivity. These modifications are directly responsible for the modulation of the $\mathrm{THz}$ signal in the range of $2-10 \mathrm{THz}$. From this permittivity model, we are able to calculate the $\mathrm{THz}$ transmission as a function of the pump irradiance (Fig. 4). These calculations fairly reproduce the experimental data with, in particular, the shift of the first transmission peak from $1 \mathrm{THz}$ to $2.5 \mathrm{THz}$. We calculate the $\mathrm{THz}$ absorption for varying pump irradiance to understand the evolution of the $\mathrm{THz}$ transmission (Fig. 5). In the range of 1-2 THz, both absorption and transmission (solid line) peaks follow the shift experienced by the ENZ frequency (dashed line). According to Eq. (2), the increase in the carrier density is responsible for the plasma frequency shift and consequently the shift of the ENZ frequency. The slab acts like a Fabry-Pérot cavity that supports resonances, which can be either symmetric or anti-symmetric with respect to the middle of the slab. When the wavelength is large compared to the slab thickness, the fundamental symmetric resonance is predominant. In this case, the amplitude of the reflected wave can be written as

$$
R=A \frac{e^{2 i k_{0} \bar{n} h}-r}{1-r e^{2 i k_{0} \bar{n} h}}
$$

where $A$ is the incident amplitude, $r=(1-\bar{n}) /(1+\bar{n})$ and $t=1+r$ are the Fresnel coefficients, and $\bar{n}=n+i \kappa$ is the complex refractive

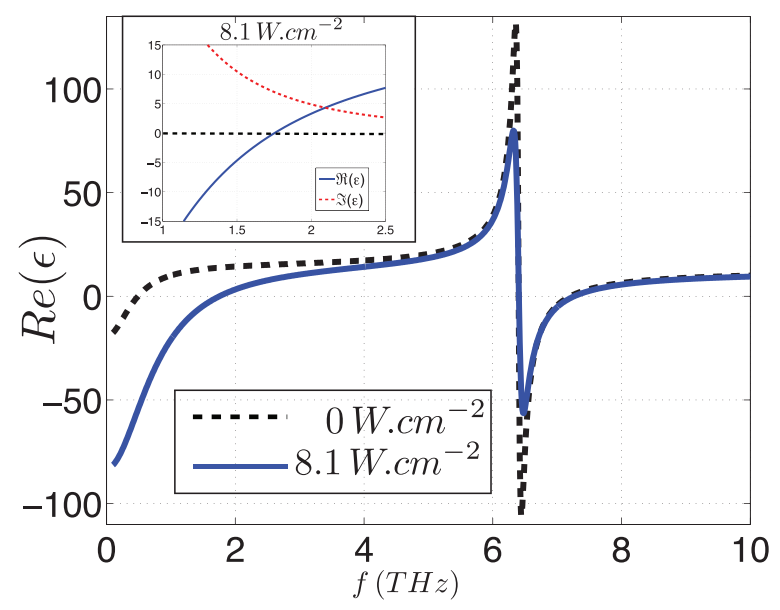

FIG. 3. Real part of the InAs permittivity for $\Phi_{0}=0 \mathrm{~W} \mathrm{~cm}^{-2}$ (dashed curve) and $\Phi_{0}=8.1 \mathrm{~W} \mathrm{~cm}^{-2}$ (solid curve). The inset shows the real and imaginary parts of the permittivity around the plasma frequency for $\Phi_{0}=8.1 \mathrm{~W} \mathrm{~cm}^{-2}$.

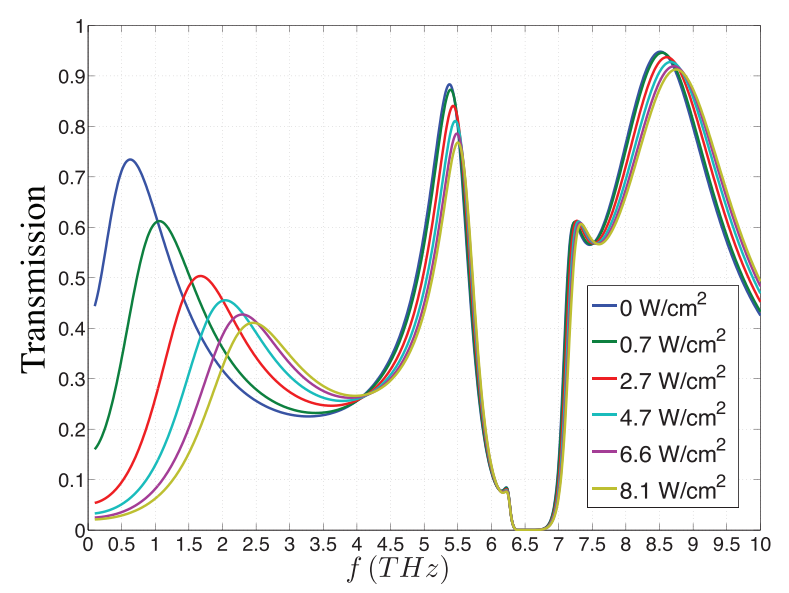

FIG. 4. Theoretical transmission spectra for increasing pump irradiance, $\Phi_{0}:\{0,0.7,2.7,4.7,6.6,8.1\} \mathrm{W} \mathrm{cm}^{-2}$.

index of InAs. The absorption of the THz wave is maximal when the reflection is null, which leads to a reflection coefficient of zero. After some analytical derivations, the thickness of the slab that satisfies this criterion is

$$
h=\frac{\lambda}{2 \pi n} \arctan \left(\frac{2 \kappa}{n^{2}+\kappa^{2}-1}\right) .
$$

At the ENZ frequency, the refractive index and absorption coefficients are equal and only determined by the imaginary part of the permittivity, $n_{0}=\kappa_{0}=\sqrt{\Im\left(\varepsilon_{m}\right) / 2}$ (see the inset of Fig. 3). In our case, $\kappa_{0}$ is larger than one, which allows us to evaluate the optimal InAs thickness $h_{0} \approx \lambda /\left(\pi \Im\left(\varepsilon_{m}\right)\right)$. From Eq. (1), we estimate that the optimal thickness is about 5 to $10 \mu \mathrm{m}$ for a carrier density between $10^{15} \mathrm{~cm}^{-3}$ and $10^{16} \mathrm{~cm}^{-3}$. The photogenerated ENZ material enhances the $\mathrm{THz}$ absorption around $1 \mathrm{THz}$ within a $\lambda / 50$ thick InAs slab. In agreement with the result of Fig. 5, the frequency shift of the THz transmission

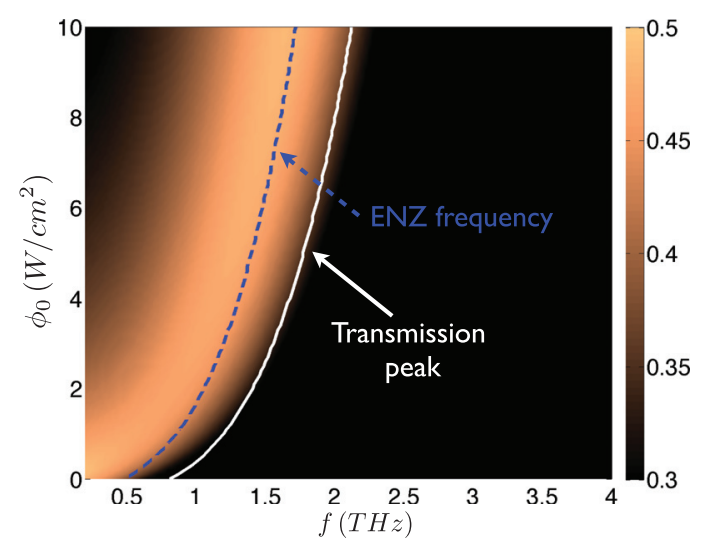

FIG. 5. Theoretical absorption as a function of the $\mathrm{THz}$ frequency and the irradiance. The dashed line represents the position of the plasma frequency, and the solid line corresponds to the transmission peak. 
originates, hence, from this ENZ absorption mechanism that occurs close to the plasma frequency, which is optically tuned.

To evaluate the dynamic performances of the InAs slab for $\mathrm{THz}$ amplitude modulation, we used the experimental setup shown in Fig. 6 and described in the figure caption. The principle of the measurements consists of the observation of the transmitted $\mathrm{THz}$ spectrum through the InAs slab illuminated by a laser beam sinusoidally modulated at increasing frequencies $f_{m}$. The laser beam irradiance is modulated by an acousto-optic modulator that attains a maximal value of $2.5 \mathrm{~W} \mathrm{~cm}^{-2}$. This optical excitation leads to a dynamic behavior of the permittivity that can be written as $\varepsilon(t)=\varepsilon+\Delta \varepsilon\left(f_{m}\right) \cos \left(2 \pi f_{m} t\right)$. The amplitude of the perturbation $\Delta \varepsilon$ depends on the efficiency of the photogeneration process, which is limited by the recombination time $\tau_{r}$. The transfer function of the photogenerated carrier density corresponds to a firstorder low-pass filter $1 / \sqrt{1+\left(2 \pi f_{m} \tau_{r}\right)^{2}}$. The oscillating behavior of the permittivity value leads to an amplitude modulation of the $\mathrm{THz}$ signal, and therefore, sidebands at $f_{0} \pm f_{m}$ appear in the THz spectrum as shown in the inset of Fig. 7 for $f_{m}=5 \mathrm{kHz}$ and for an incident $\mathrm{THz}$

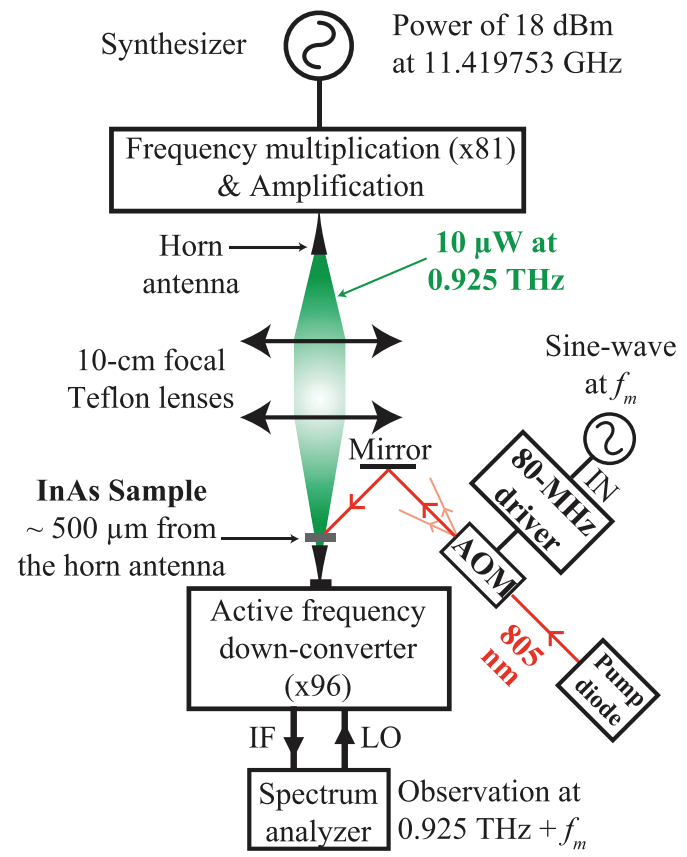

FIG. 6. Experimental setup implemented to study the dynamic $\mathrm{THz}$ transmission at $0.925 \mathrm{THz}$ through a $6 \mu \mathrm{m}$-thick InAs slab photo-excited by a $808 \mathrm{~nm}$ laser pump. This $500 \mathrm{~mW}$ laser has an amplitude modulated by a $80 \mathrm{MHz}$ acousto-optic modulator (AOM). The THz source consists of a frequency-multiplied chain (from VDI Inc.) driven by a synthesizer. The waveguide output permits the propagation of frequencies from 0.75 to $1.10 \mathrm{THz}$ with an output power close to $10 \mu \mathrm{W}$. A horn antenna is used at the waveguide output to reduce diffraction, and a couple of 10$\mathrm{cm}$-focal-length Teflon lenses is used to focus the $\mathrm{THz}$ signal into a $\mathrm{THz}$ heterodyne detector. This detector consists of an active frequency down-converter chain (from RPI Inc.) driven by the local oscillator of an electrical spectrum analyzer. Thanks to a multiplication factor of $96 \times$, the local oscillator mixes with the incoming $\mathrm{THz}$ signal at the front-end Schottky diode of the down-converter chain, thus producing a low-intermediate frequency that is detected by the spectrum analyzer. The input THz power is collected with a horn antenna. The InAs slab is located as close as possible (about $500 \mu \mathrm{m}$ ) in front of the receiver horn antenna. signal of frequency $f_{0}=0.925 \mathrm{THz}$. Remark that high frequency modulation of the permittivity is responsible for non-reciprocal behavior of light in current photonic devices. ${ }^{35,36}$ The cut-off frequency is measured by monitoring the amplitude of the satellite $\mathrm{THz}$ signal at the frequency $f_{0}+f_{m}$ as the modulation frequency of the pump $f_{m}$ is increased (see the inset of Fig. 7). The accuracy of the spectrum measurement observed using a frequency-swept analyzer is strongly degraded by the frequency noise related to the extreme frequency multiplication factors that are involved at both the source $(\times 81)$ and detection $(\times 96)$ sides. Therefore, for each modulation frequency, we acquired 20 spectra centered at $f_{0}+f_{m}$ with a span of $1 \mathrm{kHz}$ and only retained the maximum amplitude in a post-processing analysis.

The transfer function of the InAs slab is extracted from these measurements, after being corrected by the AOM transfer function that was preliminarily measured (Fig. 7). According to the photogeneration dynamics, the $\mathrm{THz}$ response of the InAs film can be fitted (red curve) by a first-order low-pass filter whose experimental cut-off frequency is $2 \mathrm{MHz}$. We remark that the transfer function drops below the fit at high modulation frequencies (above few $\mathrm{MHz}$ ) since the average pump irradiance reduces as the modulation frequency increases.

In conclusion, we have demonstrated that an ENZ absorption effect achieved in a sub-wavelength InAs slab leads to a high modulation of the $\mathrm{THz}$ signal. In the continuous regime, the modulation depth attains $90 \%$ at $1 \mathrm{THz}$. Beyond this resonant ENZ modulation, the $\mathrm{THz}$ signal is also modulated in a broadband of frequency from 2 to $10 \mathrm{THz}$ at the Fabry-Pérot resonances. This THz modulator is optically actuated by a laser pump of irradiance lower than $10 \mathrm{~W} \mathrm{~cm}^{-2}$ and works at room temperature. In the dynamic regime, the optical actuation is demonstrated to be efficient until a frequency of $2 \mathrm{MHz}$. These theoretical and experimental results open up possibilities for a
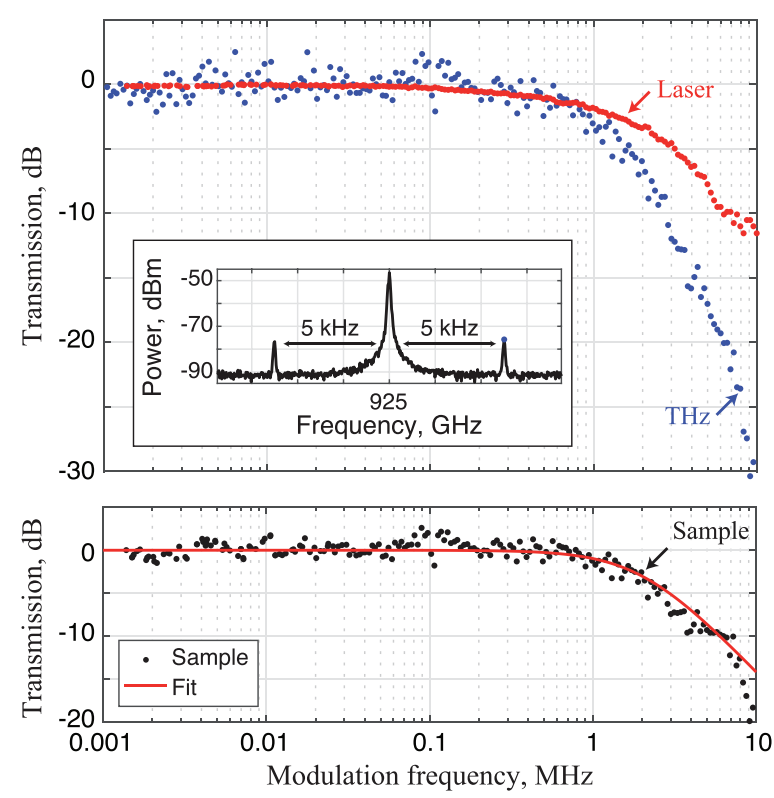

FIG. 7. The transfer function of the InAs slab: the dots correspond to the experimental data and the solid line to their fit with a first-order low pass filter. The inset of the figure shows the $\mathrm{THz}$ spectrum measured using a $10 \mathrm{~Hz}$ resolution bandwidth for a laser amplitude modulation frequency of $f_{m}=5 \mathrm{kHz}$. 
new concept of pure phase/amplitude $\mathrm{THz}$ spatial modulator for single-pixel THz imaging.

This work was partially funded by the French Investment for the Future program (EquipEx EXTRA, No. ANR 11-EQPX-0016). This work was funded by the French Labex IMOBS3.

\section{DATA AVAILABILITY}

The data that support the findings of this study are available from the corresponding author upon reasonable request.

\section{REFERENCES}

${ }^{1}$ S. Dhillon, M. Vitiello, E. Linfield, A. Davies, M. C. Hoffmann, J. Booske, C. Paoloni, M. Gensch, P. Weightman, G. Williams et al., "The 2017 terahertz science and technology roadmap,” J. Phys. D: Appl. Phys. 50, 043001 (2017).

${ }^{2}$ Y.-S. Lee, Principles of Terahertz Science and Technology (Springer Science \& Business Media, 2009), Vol. 170.

${ }^{3}$ J. F. Federici, B. Schulkin, F. Huang, D. Gary, R. Barat, F. Oliveira, and D. Zimdars, "Thz imaging and sensing for security applications-explosives, weapons and drugs," Semicond. Sci. Technol. 20, S266 (2005).

${ }^{4} \mathrm{M}$. Tonouchi, "Cutting-edge terahertz technology," Nat. Photonics 1, 97 (2007).

${ }^{5}$ K. Zaitsev, N. Chernomyrdin, K. Kudrin, I. Reshetov, and S. Yurchenko, "Terahertz spectroscopy of pigmentary skin nevi in vivo," Opt. Spectrosc. 119, 404-410 (2015)

${ }^{6} \mathrm{~S}$. Koenig, D. Lopez-Diaz, J. Antes, F. Boes, R. Henneberger, A. Leuther, A. Tessmann, R. Schmogrow, D. Hillerkuss, R. Palmer et al., "Wireless sub-THz communication system with high data rate," Nat. Photonics 7, 977-981 (2013).

${ }^{7}$ H.-T. Chen, A. J. Taylor, and N. Yu, "A review of metasurfaces: Physics and applications,” Rep. Prog. Phys. 79, 076401 (2016).

${ }^{8}$ K. Fan and W. J. Padilla, "Dynamic electromagnetic metamaterials," Mater. Today 18, 39-50 (2015).

${ }^{9}$ H.-T. Chen, W. J. Padilla, J. M. Zide, A. C. Gossard, A. J. Taylor, and R. D. Averitt, “Active terahertz metamaterial devices," Nature 444, 597 (2006).

${ }^{10}$ L. Wang, Y. Zhang, X. Guo, T. Chen, H. Liang, X. Hao, X. Hou, W. Kou, Y, Zhao, T. Zhou et al., "A review of thz modulators with dynamic tunable metasurfaces,” Nanomaterials 9, 965 (2019).

${ }^{11}$ R. Degl'Innocenti, S. J. Kindness, H. E. Beere, and D. A. Ritchie, “All-integrated terahertz modulators," Nanophotonics 7, 127-144 (2018).

${ }^{12} \mathrm{~S}$. Yu, X. Wu, Y. Wang, X. Guo, and L. Tong, "2D materials for optical modulation: Challenges and opportunities,” Adv. Mater. 29, 1606128 (2017).

${ }^{13}$ Q.-Y. Wen, W. Tian, Q. Mao, Z. Chen, W.-W. Liu, Q.-H. Yang, M. Sanderson, and H.-W. Zhang, "Graphene based all-optical spatial terahertz modulator," Sci. Rep. 4, 7409 (2015).

${ }^{14}$ Z. Ma, Z. Geng, Z. Fan, J. Liu, and H. Chen, "Modulators for terahertz communication: The current state of the art," Research 2019, 1.

${ }^{15}$ T. Okada and K. Tanaka, "Photo-designed terahertz devices," Sci. Rep. 1, 121 (2011).

${ }^{16} \mathrm{X}$. Wang, Z. Xie, W. Sun, S. Feng, Y. Cui, J. Ye, and Y. Zhang, "Focusing and imaging of a virtual all-optical tunable terahertz fresnel zone plate," Opt. Lett. 38, 4731-4734 (2013).
${ }^{17}$ G. Georgiou, H. Tyagi, P. Mulder, G. Bauhuis, J. Schermer, and J. G. Rivas, "Photo-generated THz antennas," Sci. Rep. 4, 3584 (2015).

${ }^{18}$ T. P. Steinbusch, H. K. Tyagi, M. C. Schaafsma, G. Georgiou, and J. G. Rivas, "Active terahertz beam steering by photo-generated graded index gratings in thin semiconductor films," Opt. Express 22, 26559-26571 (2014).

${ }^{19}$ G. Georgiou, C. Tserkezis, M. Schaafsma, J. Aizpurua, and J. G. Rivas, “Active loaded plasmonic antennas at terahertz frequencies: Optical control of their capacitive-inductive coupling," Phys. Rev. B 91, 125443 (2015).

${ }^{20}$ Y. Yang, N. Kamaraju, S. Campione, S. Liu, J. L. Reno, M. B. Sinclair, R. P. Prasankumar, and I. Brener, "Transient gaas plasmonic metasurfaces at terahertz frequencies," ACS Photonics 4, 15-21 (2017).

${ }^{21}$ C. Rizza, A. Ciattoni, L. Columbo, M. Brambilla, and F. Prati, "Terahertz optically tunable dielectric metamaterials without microfabrication,” Opt. Lett. 38, 1307-1309 (2013).

${ }^{22}$ E. Alvear-Cabezón, R. Smaali, E. Centeno, F. Gonzalez-Posada, and T. Taliercio, "Photogenerated metasurfaces at terahertz frequencies induced by a continuous-wave low pump," Phys. Rev. B 98, 035305 (2018).

${ }^{23}$ H. R. Seren, J. Zhang, G. R. Keiser, S. J. Maddox, X. Zhao, K. Fan, S. R. Bank, X. Zhang, and R. D. Averitt, "Nonlinear terahertz devices utilizing semiconducting plasmonic metamaterials," Light: Sci. Appl. 5, e16078 (2016).

${ }^{24}$ Y. Jin, S. Xiao, N. A. Mortensen, and S. He, "Arbitrarily thin metamaterial structure for perfect absorption and giant magnification," Opt. Express 19, 11114-11119 (2011).

${ }^{25}$ S. Feng and K. Halterman, "Coherent perfect absorption in epsilon-near-zero metamaterials," Phys. Rev. B 86, 165103 (2012).

${ }^{26}$ T. S. Luk, S. Campione, I. Kim, S. Feng, Y. C. Jun, S. Liu, J. B. Wright, I. Brener, P. B. Catrysse, S. Fan et al., "Directional perfect absorption using deep subwavelength low-permittivity films," Phys. Rev. B 90, 085411 (2014).

${ }^{27}$ S. Campione, I. Brener, and F. Marquier, "Theory of epsilon-near-zero modes in ultrathin films," Phys. Rev. B 91, 121408 (2015).

${ }^{28}$ J. Park, J.-H. Kang, X. Liu, and M. L. Brongersma, "Electrically tunable epsilonnear-zero (ENZ) metafilm absorbers,” Sci. Rep. 5, 15754 (2015).

${ }^{29}$ A. Anopchenko, L. Tao, C. Arndt, and H. W. H. Lee, "Field-effect tunable and broadband epsilon-near-zero perfect absorbers with deep subwavelength thickness," ACS Photonics 5, 2631-2637 (2018).

${ }^{30}$ Y. C. Jun, J. Reno, T. Ribaudo, E. Shaner, J.-J. Greffet, S. Vassant, F. Marquier, M. Sinclair, and I. Brener, "Epsilon-near-zero strong coupling in metamaterialsemiconductor hybrid structures," Nano Lett. 13, 5391-5396 (2013).

${ }^{31}$ S. Collin, "Nanostructure arrays in free-space: Optical properties and applications," Rep. Prog. Phys. 77, 126402 (2014).

${ }^{32}$ R. Smaali, F. Omeis, A. Moreau, T. Taliercio, and E. Centeno, "A universal design to realize a tunable perfect absorber from infrared to microwaves," Sci. Rep. 6, 32589 (2016).

${ }^{33}$ T. Taliercio and P. Biagioni, "Semiconductor infrared plasmonics," Nanophotonics 8, 949 (2019).

${ }^{34}$ G. C. Desalvo, R. Kaspi, and C. A. Bozada, "Citric acid etching of $\mathrm{GaAs}_{\mathrm{x}} \mathrm{Sb}_{\mathrm{x}}$, $\mathrm{AI}_{0.5} \mathrm{Ga}_{0.5} \mathrm{Sb}$, and InAs for heterostructure device fabrication," J. Electrochem. Soc. 141, 3526 (1994).

${ }^{35}$ D. L. Sounas and A. Alù, "Non-reciprocal photonics based on time modulation," Nat. Photonics 11, 774-783 (2017).

${ }^{36}$ K. Fang, Z. Yu, and S. Fan, "Photonic aharonov-bohm effect based on dynamic modulation," Phys. Rev. Lett. 108, 153901 (2012). 